\title{
A Rapid and Effective Method to Prolong Pain Relief in Sheep Using Bupivacaine and Intralipid ${ }^{\circledR}$ Combination
}

\author{
Farzin Sahebjam ${ }^{1 *}$, Preet Mohinder Singh ${ }^{2}$ and John Paul \\ Chambers $^{2}$ \\ ${ }^{1}$ School of Veterinary Science, Massey University, New Zealand \\ ${ }^{2}$ Animal Welfare Science and Bioethics Centre (AWSBC), School of Veterinary Science, \\ Massey University, Palmerston North, New Zealand
}

Research Article

Volume 4 Issue 3

Received Date: July 24, 2019

Published Date: August 16, 2019

DOI: $10.23880 /$ oajvsr-16000185

*Corresponding author: Farzin Sahebjam, School of Veterinary Science, Massey University, University Ave, Palmerston North, 4474, New Zealand, Email: F.Sahebjam@massey.ac.nz

\section{Abstract}

Background: To compare the duration of action of a local anesthetic block using a lipid formulation of bupivacaine to the commercially available aqueous formulation. Bupivacaine $0.5 \%$ was mixed with an equal volume of either lipid emulsion (Intralipid, Fresenius Kabi) or normal saline resulting in a final concentration of $0.25 \%$ bupivacaine. Eighteen sheep were administered a nerve block of either control or treatment at the metacarpal region of each forelimb to compare the efficacy of the injected formulations. The nociceptive test was determined by applying a blunt noxious stimulus to the foot below the nerve block at multiple time intervals until the sheep responded by withdrawing its foot. The person assessing the response to the noxious stimulus was blinded to the treatment.

Results: The Intralipid formulation significantly extended the duration of the nerve block compared to the control group. The mean analgesic period (mean $\pm \mathrm{SD}$ ) in the control legs was $4.23 \pm 1.8 \mathrm{hr}$. compared to $5.81 \pm 1.78 \mathrm{hr}$. in the Intralipid injected legs ( $\mathrm{p}=0.013)$.

Conclusions: In conclusion, an Intralipid®-based formulation provided a more prolonged duration of local anesthesia after nerve blocks in the sheep metacarpal region compared to aqueous bupivacaine.

Keywords: Bupivacaine; Sheep; Sustained; Release; Intralipid ${ }^{\circledR}$; Pain; Animal welfare

\section{Summary}

The proposed technique offers a more economical and practical method to prolong the pain relief in farm animals specifically sheep which was our animal model. Bupivacaine and Intralipid ${ }^{\circledR}$ emulsion can be mixed in a syringe by vigorous immediately before its injection under the skin. Application of a product with increased duration of action will increase animal welfare and comfort by minimizing suffering from pain induced by trauma, surgery or lameness, and reduce the need for repeated animal handling.

\section{Introduction}

Effective pain management is key to improve the welfare of sheep in pain but certain barriers such as; difficulty in recognition of pain, lack of knowledge of the 
use of analgesic and local anesthetic agents, the relatively high cost of the treatments and the possibility of residues in meat or milk, make pain management difficult in sheep. Moreover, there are no registered analgesics or local anesthetics for sheep, and they are used off-label in sheep, which exacerbates the situation [1]. An economical solution for providing safe and effective pain relief to sheep is the administration of local anesthetics around the site of injury by blocking the afferent nerve fibers. The use of long-lasting local anesthetics can mitigate the problem of repeated handling and administration of the local anesthetic agent.

Bupivacaine is an amide (aminoacyl aniline) local anesthetic commonly used in both human and veterinary clinical practice [2]. In sheep, the onset of action was within five minutes after a metacarpal block and lasted for 110 minutes [3]. A slow-release formulation of bupivacaine has also been shown to produce longer blockade with lower plasma levels than the aqueous solution in sheep brachial plexus block [4].

In comparison to other local anesthetics, bupivacaine has a longer duration of action. It is also cardiotoxic when given in higher doses to prolong its duration of action up to $20 \mathrm{hr}$ [5]. Since the side effects are related to plasma concentration following systemic absorption but nerve blockade is related to the concentration at the neuron, one strategy to increase the duration of action and reduce side effects is limiting its absorption from the site of injection.

Intralipid is a commercial injectable soybean oil emulsion designed for parenteral nutrition which strongly interacts with bupivacaine [6]. It has been shown to be useful in the treatment of bupivacaine toxicity in dogs and rats, presumably by binding free bupivacaine $[7,8]$. This ability to bind to bupivacaine may help in prolonging the duration of action of local anesthesia.

Our study aimed to test the efficacy of a simple lipid emulsion formulation of bupivacaine in sheep to prolong the duration of action. Our hypothesis was that the lipid formulation would provide analgesia of longer duration than the commercial aqueous formulation in a simple nerve block.

\section{Materials and Methods}

\section{Bupivacaine Formulations}

Intralipid ${ }^{\circledR}$ (Fresenius Kabi, Australia), a mixture of $10 \%$ soybean oil, $1.2 \%$ egg yolk phospholipids, $2.25 \%$ glycerine, and water for injection was vortex mixed with an equal volume of bupivacaine (Marcain ${ }^{\circledR}$ 0.5\%, AstraZeneca, New Zealand). The control injection was bupivacaine, and normal saline mixed similarly.

\section{Animals}

18 (6 rams and three ewes) Romney-cross mature sheep (30 to $60 \mathrm{~kg}$ ) were randomly selected for this study. They were kept on pasture at Massey University research farms with ad libitum access to water and with supplementary feed provided as required.

The sheep were acclimatized to handling as they were frequently used for teaching and low-impact research. A veterinarian clinically examined all the sheep before enrolment. The number of sheep was chosen to give a power of above $80 \%$ with G-power software program (Heinrich-Heine-Universität Düsseldorf, Germany). The study was approved by the Massey University Animal Ethics Committee (protocol 16/68).

\section{Study Design}

Sheep were manually and humanely restrained (by positioning them on their rump and holding the elbows in extension, thereby gently preventing them from moving the forefeet). The metacarpal region proximal to the coronary band was clipped (No. 40 surgical blades). The clipped area was washed and scrubbed with warm water and soap and then rinsed and dried with a clean cloth. 18 sheep received bupivacaine hydrochloride diluted in normal saline (50:50) as the control group and bupivacaine-Intralipid ${ }^{\circledR}(50: 50)$ as the treatment group either in their right and left forelimb. Each sheep received bupivacaine hydrochloride injectable formulation $(0.5 \%)$ and bupivacaine/Intralipid $\AA$ formulations randomly on either right or left forelimb. The forelimb nerve block was placed at the level of the accessory digits with three subcutaneous $(\mathrm{S} / \mathrm{C})$ injections $(1 \mathrm{~mL}$ on the cranial, $2 \mathrm{~mL}$ on the medial and $1 \mathrm{~mL}$ on the caudal side for both groups) using a $22 \mathrm{G}$ needle. The lateral aspect of each foot was intact without any injections, and it was left as a control for pain assessment to check whether the pressure was enough to cause pain or not. Therefore, the medial (locally anaesthetized) and lateral aspect (no local anesthesia) of every forefoot was assessed back and forth for better consistency. The person who injected the solutions was not later involved in pain assessment. This operator recorded which solution was injected in each foot and informed the pain assessor at the end of the experiment. The pain assessor was blinded (was not aware of what was injected and where it was injected) to reduce the study error and bias. 


\section{Nerve Block Technique}

The medial and lateral proper palmar digital nerves and medial and lateral proper dorsal digital nerves were blocked by injecting the bupivacaine solutions $(1 \mathrm{~mL})$ at three different sites (cranial, medial and caudal aspects). The injection sites were in a horizontal line at the level of dew claw, i.e., the level of first phalangeal bone and the nerves which were targeted for injection were proper dorsal digital nerves (medial and lateral proper palmar digital nerve and medial and lateral proper dorsal digital nerve). The injection areas were located and injected almost $2 \mathrm{~cm}$ above the coronary band in all animals (Figure 1).

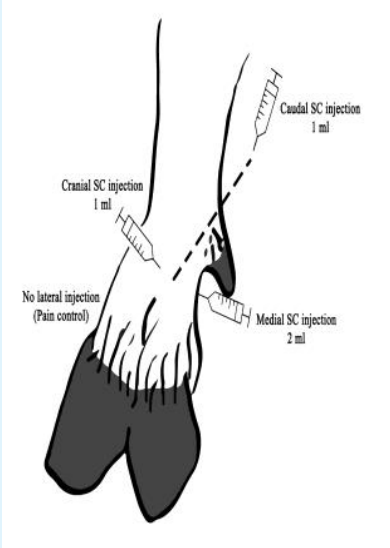

Figure 1: The schematic image of injection sites in cranial and medial aspects (injection of the caudal aspect is shown by the dashed line). The lateral side of the sheep forefeet (carpi) was considered as a control for pain assessment and no injection of bupivacaine or our treatment $=$ was carried out on that side.

\section{Pain Assessment and Data Collection}

The person assessing the pain was unaware of which formulation had been injected in each foot. Fifteen minutes after the injection, the pain was assessed by sliding the tip of a blunt hemostat on the bone where nerves pass against the skin over the P2 bone on the craniomedial aspect of the feet. Failure to respond to this noxious stimulus was considered as adequate local anesthesia.

\section{Statistical Analysis}

Normality of the distribution between groups was assessed with the Kolmogorov-Smirnoff (KS) test, and it was normal. Following this, the parametric test (Student's t-test) was used with SPSS statistical analysis program (version 2017), and the p-value was measured between the treatment and control: Intralipid®-based formulation and the control group (bupivacaine mixed with normal saline). After the analysis, the $p$-value of $<0.05$ was considered as significant. Finally, diagrams and graphs were created using Prism ${ }^{\circledR}$ Graph Pad software (version 2017).

\section{Results}

The power of the study (1- $\beta$ error probability) was calculated, and it was 0.82 , $\alpha$ error probability was 0.05 , effect size d was 1.018631, critical t was 2.036933, and non-centrality parameter $(\delta)$ was 2.969794 . These results prove that the selected sample size was sufficient to render reliable results in the study.

All eighteen sheep successfully completed the study. There was a significant increase in the duration of local anesthesia with the Intralipd $₫$-based formulation of bupivacaine compared to the control leg which was only bupivacaine diluted in normal saline (50:50). The mean analgesic period (mean \pm SD) in the control legs was $4.23 \pm 1.8 \mathrm{hr}$. compared to $5.81 \pm 1.78 \mathrm{hr}$. in the legs which were blocked by Intralipid-bupivacaine $(\mathrm{p}=0.013)$ (Figure 2).

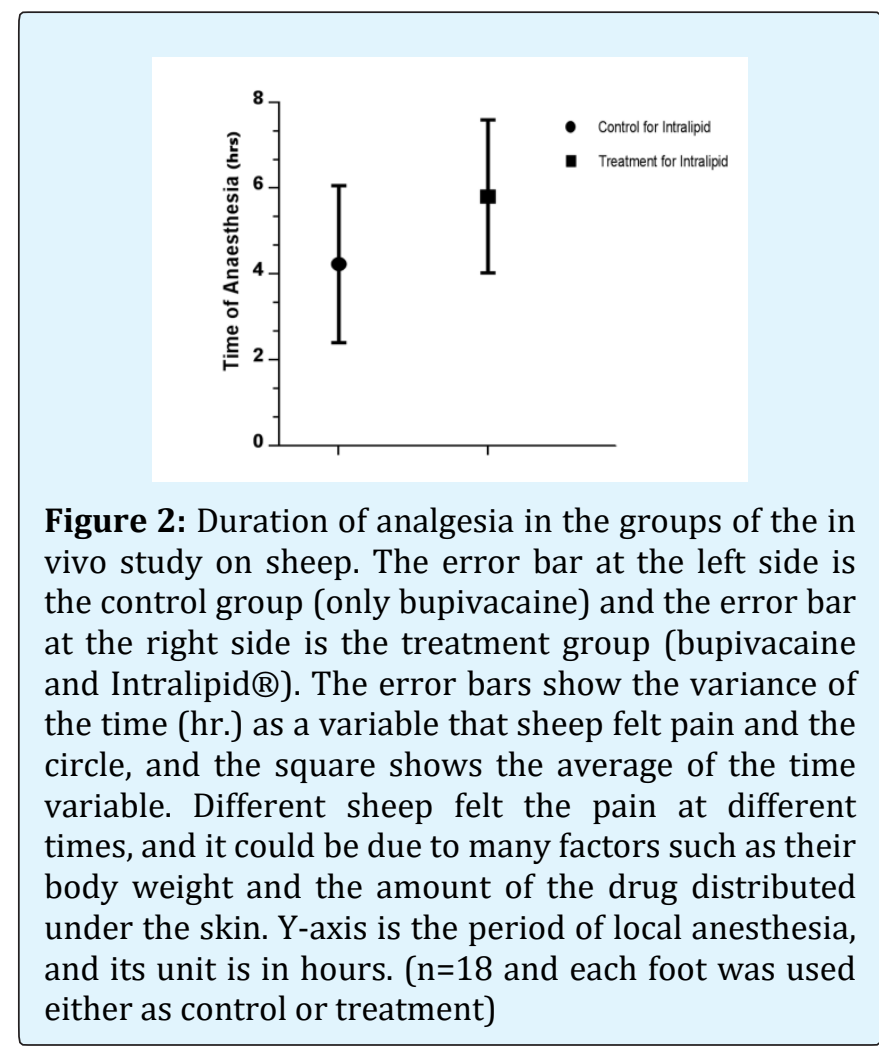




\section{Open Access Journal of Veterinary Science \& Research}

\section{Discussion}

The objective of this study was to develop a sustainedreleased bupivacaine formulation which can provide local anesthesia for a longer duration compared to the current commercially available formulation. The short duration and side effects of local anesthetics have always been a challenge for veterinarians [9]. Therefore, in the current study, a simple and cheaper method of mixing bupivacaine in with Intralipid emulsion was investigated to increase the duration of action of bupivacaine.

Intralipid is able to entrap bupivacaine molecules, presumably by dissolution in the soya bean oil, resulting in their slower release [6]. The duration of local anesthesia provided by Intralipid bupivacaine was significantly longer than bupivacaine hydrochloride aqueous solution. Intralipid interactions with bupivacaine are already known, and it is commonly used intravenously to treat bupivacaine toxicity, in particular, cardiotoxicity $[6,10]$. Intralipid has a high affinity to lipophilic substances, and it entraps these molecules in circulation by creating a significant lipid phase in plasma "lipid sink theory" [7]. In this theory, it is believed that Intralipid@ separates the bupivacaine molecules from the aqueous tissue phase. Since bupivacaine is highly lipophilic as compared to most commonly used local anesthetics, Intralipid $\AA$ interacts with its molecules strongly [11,12]; therefore, the availability of free molecules decreases (around 88\%) [6]. Provided the Intralipid stays around the nerve, the bupivacaine should continue to be slowly released, resulting in a longer duration of action.

In this study, the Intralipid $®$-based formulation showed a significant prolongation of local anesthesia of $1.58 \pm 1.5 \mathrm{hr}$. more than the control group. This formulation of bupivacaine extended the period of local anesthesia significantly compared to its control group.

This study has a number of limitations. First, plasma concentrations of bupivacaine were not measured for pharmacokinetics since the duration of pain relief was the main focus of our study. The other limitation was the pain assessment technique. Pain assessment was conducted in restrained sheep. To restrain the sheep, they were positioned on their rumps and held with forelimbs extended.

Consequently, the pain assessor was in control of when pain assessments were performed. Handlers actively tried to keep the animals as calm and as minimally agitated as possible; all sheep were kept indoors, and only water was available for the duration of the experiments. All the experiment sheep were housed together in the one enclosure, and they could easily interact with each other even while individual animals were being assessed.

The craniolateral region slightly above the coronary band was used to stimulate pain as a control for pain assessment. Thus, when the pain was present in the craniolateral region and abolished in the craniomedial region, this was confirmation of an effective nerve block. Pain response was checked by performing the same technique and pressing on the nerve sliding on the bone. Surprisingly, the craniolateral had more sensitivity to respond to almost similar pressure on the nerve by the pain assessor. That was the reason why the craniolateral area was spared as the control area for pain assessment. Also, sliding on the nerve while pressing adjacent to the bone was more effective than solely pressing the tip of the hemostat and it caused a more accurate response. For better control of the pressure, the index finger was fixed in the tip of the hemostat to keep the pressure consistent. This process was repeated every hour until the sheep responded by withdrawing its foot and that was recorded as the cut-off point for the duration of the local anesthesia. Prior to the aforementioned experimental study, several weeks were dedicated solely for the assessment of reaction to the noxious stimulus and the reaction to stress and other environmental factors. The limb retraction towards the response to the noxious stimulus was very abrupt and quick as compared to other reactions, and this was only seen in actual pain. The sheep that were enrolled in the actual study were different from the sheep in which pain assessment validation was performed by the person who was responsible for detecting all the pain reactions. The noxious stimulus was applied for three separate times for certainty of the pain reaction and to reduce any subjective decision.

The sheep did not always respond with a definitive pain reaction, and thus it took a few sessions in order to understand when the limb was being retracted as a result of pain, as compared to other factors such as stress and noise. The only reaction which was considered a true response was quick limb withdrawal after applying pressure just above the coronary band with a blunt-tip hemostat. This study mainly focused on when the sheep started to feel pain, and the amount of pressure and intensity was not the objective of the study. "Yes" as feeling pain and "No" as no pain was recorded to be able to monitor the period of local anesthesia. Validation of the application of noxious stimulus and pain assessment technique helped ensure that the results obtained were less subjective and more reliable. 


\section{Open Access Journal of Veterinary Science \& Research}

Intralipid $R$ was used as it was a commercially available preparation. In further studies, various oil-based compounds and excipients with different concentrations should be investigated to compare their results. More information is needed to understand the interactions of bupivacaine (and other local anesthetics) with various lipids.

\section{Conclusion}

In conclusion, Intralipid $₫$ was successful in significantly prolonging the action of local anesthesia (mean \pm SD $1.58 \pm 1.5 \mathrm{hr}$.) after perineural injections in the metacarpophalangeal regions of the sheep, compared to the control group. No side effects were seen with this formulation. Therefore, Intralipid® mixed with bupivacaine should provide a longer duration of pain relief. This simple formulation may be useful in farm animals in procedures such as tail docking and disbudding and provide an interesting avenue for further research.

\section{Acknowledgments}

I would like to express the deepest appreciation to the following:

Associate Professor Paul Chambers, for his expert advice and encouragement throughout this challenging project. Dr. Preet Singh, for his kind mentorship and brilliance in helping me through the research process. Mr. Antony Jacob, Mrs. Erin Willson, Mr. Gary Sparrow and Mr. Mike Reilly for their valued support and aid, especially in the practical aspect of this research project.

\section{Declarations}

- Ethics Approval and Consent to Participate: The study was approved by the Massey University Animal Ethics Committee (protocol 16/68) located at the School of Veterinary Science, Massey University, Palmerston North, New Zealand.

- Conflict of Interests: No conflict of interest was involved in this study.

- Funding: No other organization funded this project and all the project was performed at the School of Veterinary Science, Massey University, Palmerston North, New Zealand.

- Authors' Contributions: FS performed majority of procedure such as laboratory work and animal study. PMS acted as the second supervisor of the thesis and study and helped in the animal study particularly in laboratory work and injections in the animals. JPC was the main supervisor of the study, and he supervised all the procedure in addition to involving in the animal study.

\section{References}

1. Lizarraga I, Chambers JP (2012) Use of analgesic drugs for pain management in sheep. The New Zealand Veterinary Journal 60(2): 87-94.

2. Mather LE, Rutten AJ, Plummer JL (1994) Pharmacokinetics of bupivacaine enantiomers in sheep: influence of dosage regimen and study design. Journal of Pharmacokinetics and Biopharmaceutics 22(6): 481-498.

3. Lizarraga I, Janovyak E, Beths T (2013) Comparing lidocaine, bupivacaine and a lidocaine-bupivacaine mixture as a metacarpal block in sheep. Veterinary Journal 197(2): 515-518.

4. Estebe JP, Le Corre $\mathrm{P}, \mathrm{Du}$ Plessis L, Chevanne F, Cathelineau G, et al. (2001) The pharmacokinetics and pharmacodynamics of bupivacaine-loaded microspheres on a brachial plexus block model in sheep. Anesthesia \& Analgesia 93(2): 447-455.

5. Sweetman SC (2009) Martindale - The complete drug reference, 36 th $(E d n)$, Pharmaceutical Press, London, pp: 1850-1856.

6. Laine J, Lokajová J, Parshintsev J, Holopainen JM, Wiedmer SK (2010) Interaction of a commercial lipid dispersion and local anesthetics in human plasma: implications for drug trapping by "lipid-sinks." Analytical and Bioanalytical Chemistry 396(7): 25992607.

7. Weinberg G, Ripper R, Feinstein DL, Hoffman W (2003) Lipid emulsion infusion rescues dogs from bupivacaine-induced cardiac toxicity. Regional Anesthesia and Pain Medicine 28(3): 198-202.

8. Weinberg G, Lin B, Zheng S, Di Gregorio G, Hiller D, et al. (2010) Partitioning effect in lipid resuscitation: further evidence for the lipid sink. Critical Care Medicine 38(11): 2268-2269.

9. Coetzee J (2013) Pain Management. Veterinary Clinics of North America. Food Animal Practice 29(1): 11-12.

10. Mccutchen T, Gerancher JC (2008) Early Intralipid® therapy may have prevented bupivacaine-associated 


\section{Open Access Journal of Veterinary Science \& Research}

cardiac arrest. Regional Anesthesia and Pain Medicine 33(2): 178-180.

11. Muhonen J, Holopainen JM, Wiedmer SK (2009) Interactions between local anesthetics and lipid dispersions studied with liposome electrokinetic capillary chromatography. Journal of Chromatography A 1216(15): 3392-3397.
12. Doenicke AW, Roizen MF, Rau J, Kellermann W, Babl J (1996) Reducing pain during propofol injection: the role of the solvent. Anesthesia \& Analgesia 82: 472474. 